Article

\title{
On the Bicomplex Generalized Tribonacci Quaternions
}

\author{
Can Kızılateș ${ }^{1, *(1)}$, Paula Catarino ${ }^{2}(\mathbb{C})$ and Naim Tuğlu ${ }^{3}$
}

1 Department of Mathematics, Faculty of Art and Science, Zonguldak Bülent Ecevit University, 67100 Zonguldak, Turkey

2 Department of Mathematics, University of Trás-os-Montes e Alto Douro, Quinta de Prados, 5001-801 Vila Real, Portugal; pcatarin@utad.pt

3 Department of Mathematics, Faculty of Science, Gazi University, Teknikokullar, 06500 Ankara, Turkey; naimtuglu@gazi.edu.tr

* Correspondence: cankizilates@gmail.com

Received: 15 December 2018; Accepted: 10 January 2019; Published: 14 January 2019

Abstract: In this paper, we introduce the bicomplex generalized tribonacci quaternions. Furthermore, Binet's formula, generating functions, and the summation formula for this type of quaternion are given. Lastly, as an application, we present the determinant of a special matrix, and we show that the determinant is equal to the $n^{\text {th }}$ term of the bicomplex generalized tribonacci quaternions.

Keywords: bicomplex number; generalized tribonacci sequence; bicomplex generalized tribonacci quaternion

MSC: 11B39; 20G20; 11R52

\section{Introduction}

Corrado Segre introduced bicomplex numbers in 1892. The bicomplex numbers are defined by four elements $1, i, j, i j$, where $i, j$ and $i j$ satisfy the properties:

$$
i^{2}=-1, j^{2}=-1, i j=j i .
$$

Thus, a bicomplex number $q$ can be expressed as follows:

$$
q=q_{1}+i q_{2}+j q_{3}+i j q_{4}=q_{1}+i q_{2}+j\left(q_{3}+i q_{4}\right),
$$

where $q_{1}, q_{2}, q_{3}, q_{4} \in \mathbb{R}$.

For any $q=q_{1}+i q_{2}+j q_{3}+i j q_{4}$ and $p=p_{1}+i p_{2}+j p_{3}+i j p_{4}$, the addition, subtraction, and multiplication of the bicomplex quaternions are defined by the following:

$$
\begin{gathered}
q+p=q_{1}+p_{1}+i\left(q_{2}+p_{2}\right)+j\left(q_{3}+p_{3}\right)+i j\left(q_{4}+p_{4}\right), \\
q-p=q_{1}-p_{1}+i\left(q_{2}-p_{2}\right)+j\left(q_{3}-p_{3}\right)+i j\left(q_{4}-p_{4}\right), \\
q \times p=q_{1} p_{1}-q_{2} p_{2}-q_{3} p_{3}+q_{4} p_{4}+i\left(q_{1} p_{2}+q_{2} p_{1}-q_{3} p_{4}-q_{4} p_{3}\right) \\
+j\left(q_{1} p_{3}+q_{3} p_{1}-q_{2} p_{4}-q_{4} p_{2}\right)+i j\left(q_{1} p_{4}+q_{4} p_{1}+q_{2} p_{3}+q_{3} p_{2}\right),
\end{gathered}
$$

respectively.

Bicomplex numbers have three different conjugations as follows:

$$
q_{i}^{*}=q_{1}-i q_{2}+j q_{3}-i j q_{4}
$$




$$
\begin{aligned}
& q_{j}^{*}=q_{1}+i q_{2}-j q_{3}-i j q_{4}, \\
& q_{i j}^{*}=q_{1}-i q_{2}-j q_{3}+i j q_{4} .
\end{aligned}
$$

For more details about the bicomplex numbers, the readers can refer to [1-4].

On the other hand, the Irish mathematician William Rowan Hamilton introduced quaternions as an extension of complex numbers. A quaternion $q$ is defined in the form:

$$
q=q_{0}+q_{1} \mathbf{i}+q_{2} \mathbf{j}+q_{3} \mathbf{k}
$$

where $q_{0}, q_{1}, q_{2}, q_{3}$ are real numbers and $\mathbf{1}, \mathbf{i}, \mathbf{j}, \mathbf{k}$ are the standard orthonormal basis in $\mathbb{R}^{4}$, which satisfy the quaternion multiplication rules as:

$$
\begin{gathered}
\mathbf{i}^{2}=\mathbf{j}^{2}=\mathbf{k}^{2}=\mathbf{i j k}=-1, \\
\mathbf{i j}=\mathbf{k}=-\mathbf{j} \mathbf{i}, \mathbf{j} \mathbf{k}=\mathbf{i}=-\mathbf{k} \mathbf{j}, \mathbf{k i}=\mathbf{j}=-\mathbf{i k} .
\end{gathered}
$$

Immediately, we note that quaternions and bicomplex numbers are generalizations of complex numbers, but one of the important differences between them is that quaternions are non-commutative, whereas bicomplex numbers are commutative. Furthermore, the bicomplex quaternions can be defined by the basis $\mathbf{1}, \mathbf{i}, \mathbf{j}$ and $\mathbf{i j}$ that satisfy the rules (1).

The $n^{\text {th }}$ Fibonacci and Lucas quaternions were defined by Horadam for $n \geq 0$,

$$
\begin{aligned}
& Q_{n}=F_{n}+F_{n+1} \mathbf{i}+F_{n+2} \mathbf{j}+F_{n+3} \mathbf{k}, \\
& K_{n}=L_{n}+L_{n+1} \mathbf{i}+L_{n+2} \mathbf{j}+L_{n+3} \mathbf{k},
\end{aligned}
$$

where $F_{n}$ and $L_{n}$ denote the $n^{\text {th }}$ Fibonacci and Lucas numbers, respectively [5]. There are many generalizations of the Fibonacci, and Fibonacci-like quaternions have been studied in the literature (see for example [5-18]).

The generalized tribonacci sequence, $\left\{T_{n}=T_{n}(r, s, t ; a, b, c)\right\}_{n \geq 0}$, is defined by the following recurrence relation:

$$
T_{n}=r T_{n-1}+s T_{n-2}+t T_{n-3}
$$

where $T_{0}=a, T_{1}=b, T_{2}=c$ are arbitrary integers and $r, s, t$ are real numbers. The Binet formula for the generalized tribonacci sequence is given by the following relationship:

$$
T_{n}=\frac{A \alpha^{n}}{(\alpha-\beta)(\alpha-\gamma)}-\frac{B \beta^{n}}{(\alpha-\beta)(\beta-\gamma)}+\frac{C \gamma^{n}}{(\alpha-\gamma)(\beta-\gamma)},
$$

where $A=c-(\beta+\gamma) b+\beta \gamma a, B=c-(\alpha+\gamma) b+\alpha \gamma a$ and $C=c-(\alpha+\beta) b+\alpha \beta a$. The following are some special cases of the generalized tribonacci sequence $T_{n}(r, s, t ; a, b, c)$ :

1. for $r=s=t=1$ and $a=0, b=c=1$, the generalized tribonacci sequence $T_{n}(1,1,1 ; 0,1,1)$ becomes the tribonacci sequence $T_{n}$;

2. for $r=s=t=1$ and $a=3, b=1, c=3$, the generalized tribonacci sequence $T_{n}(1,1,1 ; 3,1,3)$ becomes the tribonacci-Lucas sequence $K_{n}$;

3. for $r=0, s=t=1$ and $a=3, b=0, c=2$, the generalized tribonacci sequence $T_{n}(0,1,1 ; 3,0,2)$ becomes the Perrin sequence $Q_{n}$;

4. for $r=0, s=t=1$ and $a=b=c=1$, the generalized tribonacci sequence $T_{n}(0,1,1 ; 1,1,1)$ becomes the Padovan (Cordonnier) sequence $P_{n}$;

5. for $r=0, s=t=1$ and $a=1, b=0, c=1$, the generalized tribonacci sequence $T_{n}(0,1,1 ; 1,0,1)$ becomes the Van der Laan sequence $R_{n}$;

6. for $r=1, s=0, t=1$ and $a=0, b=c=1$, the generalized tribonacci sequence $T_{n}(1,0,1 ; 0,1,1)$ becomes the Narayana sequence $B_{n}$; 
7. for $r=s=1, t=2$ and $a=0, b=c=1$, the generalized tribonacci sequence $T_{n}(1,1,2 ; 0,1,1)$ becomes the third-order Jacobsthal sequence $J_{n}^{(3)}$;

8. for $r=s=1, t=2$ and $a=2, b=1, c=5$, the generalized tribonacci sequence $T_{n}(1,1,2 ; 2,1,5)$ becomes the third-order Jacobsthal-Lucas sequence $j_{n}^{(3)}$;

Therefore, the generalized tribonacci sequences are the generalization of the well-known sequences, which have been studied extensively in the literature (see [19-27]).

The tribonacci quaternion sequences and some generalizations have been studied by many researchers in their papers (see for example [9]).

In [28], the authors defined the bicomplex Fibonacci and Lucas numbers. Moreover, they gave some algebraic properties of these numbers. In [29], Aydın studied the bicomplex Fibonacci quaternions. Recently, in [30], Catarino defined the bicomplex $k$-Pell quaternions and examined some properties involving these quaternions.

Motivated by the above papers, in this paper, we introduce the bicomplex generalized tribonacci quaternion, and we obtain Binet's formula, generating functions, and summation formula, as well as some other properties. We explain that the new bicomplex generalized tribonacci quaternions include the bicomplex tribonacci quaternions, the bicomplex tribonacci-Lucas quaternions, the bicomplex Perrin quaternions, the bicomplex Padovan quaternions, the bicomplex Van der Laan quaternions, the bicomplex Narayana quaternions, the bicomplex third-order Jacobsthal quaternions, and the bicomplex third-order Jacobsthal-Lucas quaternions, respectively.

\section{Bicomplex Generalized Tribonacci Quaternions}

In this section, we introduce the bicomplex generalized tribonacci quaternions.

Definition 1. The bicomplex generalized tribonacci quaternions $\left\{\mathbb{B C} T_{n}\right\}_{n=0}^{\infty}$ are defined by the following recurrence relation:

$$
\mathbb{B C} T_{n}=T_{n}+i T_{n+1}+j T_{n+2}+i j T_{n+3},
$$

where $T_{n}$ is the generalized tribonacci quaternion and $1, i, j, i j$ satisfy the rules (1).

From (2), taking into account (4), we have the recurrence relation of the bicomplex generalized tribonacci quaternions as follows:

$$
\mathbb{B C} T_{n+2}=r \mathbb{B} \mathbb{C} T_{n+1}+s \mathbb{B} \mathbb{C} T_{n}+t \mathbb{B C} T_{n-1}, \quad n \geq 1
$$

with the initial conditions $\mathbb{B C} T_{0}=a+i b+j c+i j(r c+s b+t a), \mathbb{B C C} T_{1}=b+i c+j(r c+s b+t a)+$ $i j\left(\left(r^{2}+s\right) c+(t+r s) b+r t a\right)$, and $\mathbb{B} \mathbb{C} T_{2}=c+i(r c+s b+t a)+j\left(\left(r^{2}+s\right) c+(t+r s) b+r t a\right)+i j\left(\left(r^{3}+\right.\right.$ $\left.2 r s t+t) c+\left(r^{2} s+s^{2}+r t\right) b+\left(r^{2} t+s t\right) a\right)$.

For two bicomplex generalized tribonacci quaternions $\mathbb{B} \mathbb{C} T_{n}$ and $\mathbb{B C} T_{m}$, addition, subtraction, and multiplication are defined by the following:

$$
\begin{aligned}
\mathbb{B C} T_{n} \pm \mathbb{B C} T_{m}=T_{n} \pm T_{m} & +i\left(T_{n+1} \pm T_{m+1}\right)+j\left(T_{n+2} \pm T_{m+2}\right)+i j\left(T_{n+3} \pm T_{m+3}\right), \\
\mathbb{B C} T_{n} \times \mathbb{B C C} T_{m}= & \left(T_{n} T_{m}-T_{n+1} T_{m+1}-T_{n+2} T_{m+2}+T_{n+3} T_{m+3}\right) \\
& +i\left(T_{n} T_{m+1}+T_{n+1} T_{m}-T_{n+2} T_{m+3}-T_{n+3} T_{m+2}\right) \\
& +j\left(T_{n} T_{m+2}+T_{n+2} T_{m}-T_{n+1} T_{m+3}-T_{n+3} T_{m+1}\right) \\
& +i j\left(T_{n} T_{m+3}+T_{n+3} T_{m}+T_{n+1} T_{m+2}+T_{n+2} T_{m+1}\right) \\
= & \mathbb{B C} T_{m} \times \mathbb{B C} T_{n} .
\end{aligned}
$$


The multiplication of a bicomplex generalized tribonacci quaternions by the real scalar $\lambda$ is defined as follows:

$$
\lambda \mathbb{B} \mathbb{C} T_{n}=\lambda T_{n}+i \lambda T_{n+1}+j \lambda T_{n+2}+i j \lambda T_{n+3} .
$$

Furthermore, three different conjugations for the bicomplex generalized tribonacci quaternions are presented by the following:

$$
\begin{aligned}
& \left(\mathbb{B C C} T_{n}\right)_{i}^{*}=T_{n}-i T_{n+1}+j T_{n+2}-i j T_{n+3}, \\
& \left(\mathbb{B C C} T_{n}\right)_{j}^{*}=T_{n}+i T_{n+1}-j T_{n+2}-i j T_{n+3}, \\
& \left(\mathbb{B C C} T_{n}\right)_{i j}^{*}=T_{n}-i T_{n+1}-j T_{n+2}+i j T_{n+3} .
\end{aligned}
$$

Now, we define the generating function:

$$
G F_{\mathbb{B C} T_{n}}(x)=\sum_{n=0}^{\infty} \mathbb{B} \mathbb{C} T_{n} x^{n}
$$

Theorem 1. The generating function for the bicomplex generalized tribonacci quaternions sequence is as follows:

$$
\frac{\mathbb{B C} T_{0}+\left(\mathbb{B C} T_{1}-r \mathbb{B C} T_{0}\right) x+\left(\mathbb{B C} T_{2}-r \mathbb{B} \mathbb{C} T_{1}-s \mathbb{B C} T_{0}\right) x^{2}}{1-r x-s x^{2}-t x^{3}} .
$$

Proof. Using the definition of generating function:

$$
\operatorname{GF}_{\mathbb{B C} T_{n}}(x)=\mathbb{B C} T_{0}+\mathbb{B C} T_{1} x+\cdots+\mathbb{B} \mathbb{C} T_{n} x^{n}+\cdots
$$

Multiplying both sides of (6) by $\left(1-r x-s x^{2}-t x^{3}\right)$ and using (5), we have:

$$
\left(1-r x-s x^{2}-t x^{3}\right)=\mathbb{B C} T_{0}+\left(\mathbb{B C} T_{1}-r \mathbb{B} \mathbb{C} T_{0}\right) x+\left(\mathbb{B C} T_{2}-r \mathbb{B} \mathbb{C} T_{1}-s \mathbb{B} \mathbb{C} T_{0}\right) x^{2} .
$$

Thus, the proof is completed.

Theorem 2. The Binet formula for the bicomplex generalized tribonacci quaternions is:

$$
\mathbb{B C} T_{n}=\frac{A \widehat{\alpha} \alpha^{n}}{(\alpha-\beta)(\alpha-\gamma)}-\frac{B \widehat{\beta} \beta^{n}}{(\alpha-\beta)(\beta-\gamma)}+\frac{C \widehat{\gamma} \gamma^{n}}{(\alpha-\gamma)(\beta-\gamma)}, \quad n \geq 0
$$

where $\widehat{\alpha}=1+i \alpha+j \alpha^{2}+i j \alpha^{3}, \widehat{\beta}=1+i \beta+j \beta^{2}+i j \beta^{3}$, and $\widehat{\gamma}=\gamma+i \gamma+j \gamma^{2}+i j \gamma^{3}$.

Proof. Using the definition of bicomplex generalized tribonacci quaternion and (3), we have:

$$
\begin{aligned}
\mathbb{B C} T_{n}= & \left(\frac{A \alpha^{n}}{(\alpha-\beta)(\alpha-\gamma)}-\frac{B \beta^{n}}{(\alpha-\beta)(\beta-\gamma)}+\frac{C \gamma^{n}}{(\alpha-\gamma)(\beta-\gamma)}\right) \\
& +i\left(\frac{A \alpha^{n+1}}{(\alpha-\beta)(\alpha-\gamma)}-\frac{B \beta^{n+1}}{(\alpha-\beta)(\beta-\gamma)}+\frac{C \gamma^{n+1}}{(\alpha-\gamma)(\beta-\gamma)}\right) \\
& +j\left(\frac{A \alpha^{n+2}}{(\alpha-\beta)(\alpha-\gamma)}-\frac{B \beta^{n+2}}{(\alpha-\beta)(\beta-\gamma)}+\frac{C \gamma^{n+2}}{(\alpha-\gamma)(\beta-\gamma)}\right) \\
& +i j\left(\frac{A \alpha^{n+3}}{(\alpha-\beta)(\alpha-\gamma)}-\frac{B \beta^{n+3}}{(\alpha-\beta)(\beta-\gamma)}+\frac{C \gamma^{n+3}}{(\alpha-\gamma)(\beta-\gamma)}\right)
\end{aligned}
$$

After some basic calculation, the proof is completed. 
Theorem 3. For $n \in \mathbb{N}$, the exponential generating function for the bicomplex generalized tribonacci quaternions is:

$$
\sum_{n=0}^{\infty} \mathbb{B} \mathbb{C} T_{n} \frac{x^{n}}{n !}=\frac{A \widehat{\alpha} e^{\alpha x}}{(\alpha-\beta)(\alpha-\gamma)}-\frac{B \widehat{\beta} e^{\beta x}}{(\alpha-\beta)(\beta-\gamma)}+\frac{C \widehat{\gamma} e^{\gamma x}}{(\alpha-\gamma)(\beta-\gamma)},
$$

where $\widehat{\alpha}=1+i \alpha+j \alpha^{2}+i j \alpha^{3}, \widehat{\beta}=1+i \beta+j \beta^{2}+i j \beta^{3}$, and $\widehat{\gamma}=\gamma+i \gamma+j \gamma^{2}+i j \gamma^{3}$.

Proof. By using the Binet formula for the bicomplex generalized tribonacci quaternions given in Theorem 2, we have:

$$
\begin{aligned}
\sum_{n=0}^{\infty} \mathbb{B} \mathbb{C} T_{n} \frac{x^{n}}{n !}= & \frac{A \widehat{\alpha}}{(\alpha-\beta)(\alpha-\gamma)} \sum_{n=0}^{\infty} \frac{(\alpha x)^{n}}{n !}-\frac{B \widehat{\beta}}{(\alpha-\beta)(\beta-\gamma)} \sum_{n=0}^{\infty} \frac{(\beta x)^{n}}{n !} \\
& +\frac{C \widehat{\gamma}}{(\alpha-\gamma)(\beta-\gamma)} \sum_{n=0}^{\infty} \frac{(\gamma x)^{n}}{n !} \\
= & \frac{A \widehat{\alpha} e^{\alpha x}}{(\alpha-\beta)(\alpha-\gamma)}-\frac{B \widehat{\beta} e^{\beta x}}{(\alpha-\beta)(\beta-\gamma)}+\frac{C \widehat{\gamma} e^{\gamma x}}{(\alpha-\gamma)(\beta-\gamma)} .
\end{aligned}
$$

Thus, the proof is completed.

Lemma 1. [9] (Page 239, Lemma 2.3) For the generalized tribonacci numbers, the following equation holds for:

$$
\sum_{l=0}^{n} T_{l}=\frac{1}{\delta(r, s, t)}\left(T_{n+2}+(1-r) T_{n+1}+t T_{n}+\lambda(r, s, t)\right),
$$

where $\delta(r, s, t)=r+s+t-1$ and $\lambda(r, s, t)=(r+s-1) a+(r-1) b-c$.

Theorem 4. The summation formula for the bicomplex generalized tribonacci quaternions is given by the following:

$$
\sum_{l=0}^{n} \mathbb{B} \mathbb{C} T_{l}=\frac{1}{\delta(r, s, t)}\left[\mathbb{B C} T_{n+2}+(1-r) \mathbb{B C} T_{n+1}+t \mathbb{B} \mathbb{C} T_{n}+\lambda(r, s, t)\right] .
$$

Proof. Using (4), we have:

$$
\sum_{l=0}^{n} \mathbb{B} \mathbb{C} T_{l}=\sum_{l=0}^{n} T_{l}+i \sum_{l=0}^{n} T_{l+1}+j \sum_{l=0}^{n} \mathrm{~T}_{l+2}+i j \sum_{l=0}^{n} \mathrm{~T}_{l+3} .
$$

Using the Lemma 1, the proof is completed.

\section{An Application of Bicomplex Generalized Tribonacci Quaternions in Matrices}

In this section, we give another method to obtain the $n^{\text {th }}$ term of the bicomplex generalized tribonacci quaternion sequence as the calculation of the determinant of a special matrix. For this purpose, we give the following theorem.

Theorem 5. Let $\left\{x_{n}\right\}$ be any third-order linear sequence, defined recursively as:

$$
x_{n+1}=r x_{n}+s x_{n-1}+t x_{n-2}, \quad n \geq 2
$$

with the initial values $x_{0}=a, x_{1}=b$, and $x_{2}=c$. Then, for all $n \geq 0$, 


$$
x_{n}=\left|\begin{array}{rrrrrrrr}
a & -1 & 0 & 0 & 0 & \cdots & 0 & 0 \\
b & 0 & -1 & 0 & 0 & \cdots & 0 & 0 \\
c & 0 & 0 & -1 & 0 & \cdots & 0 & 0 \\
0 & t & r & s & -1 & \cdots & 0 & 0 \\
\vdots & \ddots & \ddots & \ddots & \ddots & \ddots & \vdots & \vdots \\
0 & 0 & 0 & 0 & 0 & \ddots & s & -1 \\
0 & 0 & 0 & 0 & 0 & \ddots & r & s
\end{array}\right|_{(n+1) \times(n+1)}
$$

Proof. For the proof, we use the induction method on $n$. The equality holds for $n=0,1,2$. Namely,

$$
\begin{aligned}
& x_{0}=|a|_{1 \times 1}=a, \\
& x_{1}=\left|\begin{array}{rr}
a & -1 \\
b & 0
\end{array}\right|_{2 \times 2}=b, \\
& x_{2}=\left|\begin{array}{rrr}
a & -1 & 0 \\
b & 0 & -1 \\
c & 0 & 0
\end{array}\right|_{3 \times 3}=c .
\end{aligned}
$$

Now, suppose that the equality is true for $2<k \leq n$. Then, we can verify it for $n+1$ as follows:

$$
\begin{aligned}
& x_{n+1}=\left|\begin{array}{rrrrrrrrr}
a & -1 & 0 & 0 & 0 & \cdots & 0 & 0 & 0 \\
b & 0 & -1 & 0 & 0 & \cdots & 0 & 0 & 0 \\
c & 0 & 0 & -1 & 0 & \cdots & 0 & 0 & 0 \\
0 & t & r & s & -1 & \cdots & 0 & 0 & 0 \\
\vdots & \ddots & \ddots & \ddots & \ddots & \ddots & \vdots & \vdots & \vdots \\
0 & 0 & 0 & 0 & 0 & \ddots & s & -1 & 0 \\
0 & 0 & 0 & 0 & 0 & \ddots & r & s & -1 \\
0 & 0 & 0 & 0 & 0 & & t & r & s
\end{array}\right|_{(n+2) \times(n+2)} \\
& =s(-1)^{(n+2)+(n+2)}\left|\begin{array}{rrrrrrrr}
a & -1 & 0 & 0 & 0 & \cdots & 0 & 0 \\
b & 0 & -1 & 0 & 0 & \cdots & 0 & 0 \\
c & 0 & 0 & -1 & 0 & \cdots & 0 & 0 \\
0 & t & r & s & -1 & \cdots & 0 & 0 \\
\vdots & \ddots & \ddots & \ddots & \ddots & \ddots & \vdots & \vdots \\
0 & 0 & 0 & 0 & 0 & \ddots & s & -1 \\
0 & 0 & 0 & 0 & 0 & \ddots & r & s
\end{array}\right|_{(n+1) \times(n+1)} \\
& +(-1)(-1)^{(n+2)+(n+1)}\left|\begin{array}{rrrrrrrr}
a & -1 & 0 & 0 & 0 & \cdots & 0 & 0 \\
b & 0 & -1 & 0 & 0 & \cdots & 0 & 0 \\
c & 0 & 0 & -1 & 0 & \cdots & 0 & 0 \\
0 & t & r & s & -1 & \cdots & 0 & 0 \\
\vdots & \ddots & \ddots & \ddots & \ddots & \ddots & \vdots & \vdots \\
0 & 0 & 0 & 0 & 0 & \ddots & s & -1 \\
0 & 0 & 0 & 0 & 0 & & t & r
\end{array}\right|_{(n+1) \times(n+1)}
\end{aligned}
$$




$$
\begin{aligned}
& x_{n+1}=s x_{n}+r\left|\begin{array}{rrrrrrr}
a & -1 & 0 & 0 & 0 & \cdots & 0 \\
b & 0 & -1 & 0 & 0 & \cdots & 0 \\
c & 0 & 0 & -1 & 0 & \cdots & 0 \\
0 & t & r & s & -1 & \cdots & 0 \\
\vdots & \ddots & \ddots & \ddots & \ddots & \ddots & \vdots \\
0 & 0 & 0 & 0 & 0 & \ddots & s
\end{array}\right|_{n \times n}+\left|\begin{array}{rrrrrrr}
a & -1 & 0 & 0 & 0 & \cdots & 0 \\
b & 0 & -1 & 0 & 0 & \cdots & 0 \\
c & 0 & 0 & -1 & 0 & \cdots & 0 \\
0 & t & r & s & -1 & \cdots & 0 \\
\vdots & \ddots & \ddots & \ddots & \ddots & \ddots & \vdots \\
0 & 0 & 0 & 0 & 0 & & t
\end{array}\right|_{n \times n} \\
& =s x_{n}+r x_{n-1}+\left|\begin{array}{rrrrrrr}
a & -1 & 0 & 0 & 0 & \cdots & 0 \\
b & 0 & -1 & 0 & 0 & \cdots & 0 \\
c & 0 & 0 & -1 & 0 & \cdots & 0 \\
0 & t & r & s & -1 & \cdots & 0 \\
\vdots & \ddots & \ddots & \ddots & \ddots & \ddots & \vdots \\
0 & 0 & 0 & 0 & 0 & & t
\end{array}\right|_{n \times n} \\
& =s x_{n}+r x_{n-1}+t(-1)^{n+n}\left|\begin{array}{rrrrrrr}
a & -1 & 0 & 0 & 0 & \cdots & 0 \\
b & 0 & -1 & 0 & 0 & \cdots & 0 \\
c & 0 & 0 & -1 & 0 & \cdots & 0 \\
0 & t & r & s & -1 & \cdots & 0 \\
\vdots & \ddots & \ddots & \ddots & \ddots & \ddots & \vdots \\
0 & 0 & 0 & 0 & 0 & & -1
\end{array}\right|_{(n-1) \times(n-1)} \\
& =s x_{n}+r x_{n-1}+t x_{n-2} \text {. }
\end{aligned}
$$

Thus, the proof is completed.

Using the above theorem, we have the following result, which gives a different way to obtain the $n^{\text {th }}$ term of bicomplex generalized tribonacci quaternions.

Theorem 6. For $n \geq 0$, we have:

$$
\mathbb{B C} T_{n}=\left|\begin{array}{rrrrrrrr}
\mathbb{B} C T_{0} & -1 & 0 & 0 & 0 & \cdots & 0 & 0 \\
\mathbb{B} C T_{1} & 0 & -1 & 0 & 0 & \cdots & 0 & 0 \\
\mathbb{B C} T_{2} & 0 & 0 & -1 & 0 & \cdots & 0 & 0 \\
0 & t & r & s & -1 & \cdots & 0 & 0 \\
\vdots & \ddots & \ddots & \ddots & \ddots & \ddots & \vdots & \vdots \\
0 & 0 & 0 & 0 & 0 & \ddots & s & -1 \\
0 & 0 & 0 & 0 & 0 & \ddots & r & s
\end{array}\right|_{(n+1) \times(n+1)}
$$

Proof. By using (5) and the previous theorem, the proof can be easily shown.

\section{Conclusions}

In this paper, we defined the bicomplex generalized tribonacci quaternions. Some properties involving this sequence, including the Binet formula, generating function, exponential generating function, and the summation formula, were presented. Moreover, a special matrix whose entries are bicomplex generalized tribonacci quaternions was presented as a different way to obtain the $n^{\text {th }}$ term of the bicomplex generalized tribonacci quaternions sequence. One of the aims of this work is to obtain bicomplex quaternion versions of tribonacci or tribonacci-like sequences in the literature.

Author Contributions: All authors contributed equally to this work. All authors read and approved the final manuscript. 
Funding: This research received no external funding.

Acknowledgments: The authors would like to express their sincere gratitude to the referees for their valuable comments, which have significantly improved the presentation of this paper.

Conflicts of Interest: The authors declare no conflict of interest.

\section{References}

1. Elizarrarás-Luna, M.E.; Shapiro, M.; Struppa, M.; Vajiac, A.D.C. The bicomplex numbers. In Bicomplex Holomorphic Functions; Frontiers in Mathematics, Birkhuser: Cham, Switzerland, 2015; pp. 5-28.

2. Luna-Elizarraras, M.E.; Shapiro, M.; Struppa, D.C.; Vajiac, A. Bicomplex numbers and their elementary functions. Cubo 2012, 44, 61-80. [CrossRef]

3. Rochon, D.; Shapiro, M. On algebraic properties of bicomplex and hyperbolic numbers. Anal. Univ. Oradea Fasc. Math. 2004, 11, 71-110.

4. Segre, C. Le Rappresentazioni Reali Delle Forme Complesse e Gli Enti Iperalgebrici. Math. Ann. 1892, 40, 413-467. [CrossRef]

5. Horadam, A.F. Complex Fibonacci numbers and Fibonacci quaternions. Am. Math. Mon. 1963, 70, $289-291$. [CrossRef]

6. Catarino, P. The Modified Pell and Modified k-Pell Quaternions and Octonions. Adv. Appl. Clifford Algebr. 2016, 26, 577-590. [CrossRef]

7. Catarino, P. A note on $h(x)$-Fibonacci quaternion polynomials. Chaos Solitons Fractals 2015, 77, 1-5. [CrossRef]

8. Catarino, P. A note on certain matrices with $h(x)$-Fibonacci quaternion polynomials. J. Differ. Equ. Appl. 2016, 22, 343-351. [CrossRef]

9. Cerda-Morales, G. On a generalization for tribonacci quaternions. Mediterr. J. Math. 2017, 14, 239. [CrossRef]

10. Halici, S. On Fibonacci quaternions. Adv. Appl. Clifford Algebr. 2012, 22, 321-327. [CrossRef]

11. Horadam, A.F. Quaternion recurrence relations. Ulam Q. 1993, 2, $23-33$.

12. Iakin, A.L. Extended Binet forms for generalized quaternions of higher order. Fibonacci Q. 1981, 19, 10-13.

13. Iakin, A.L. Generalized quaternions of higher order. Fibonacci Q. 1977, 15, 343-346.

14. Iakin, A.L. Generalized quaternions with quaternion components. Fibonacci Q. 1977, 15, 225-230.

15. Iyer, M.R. A note on Fibonacci quaternions. Fibonacci Q. 1969, 7, 225-229.

16. Iyer, M.R. Some results on Fibonacci quaternions. Fibonacci Q. 1969, 7, 201-210.

17. Polatli, E. A generalization of Fibonacci and Lucas Quaternions. Adv. Appl. Clifford Algebr. 2015. [CrossRef]

18. Tasci, D.; Yalcin, F. Fibonacci-p quaternions. Adv. Appl. Clifford Algebr. 2015, 25, 245-254. [CrossRef]

19. Cerda-Morales, G. On the third-order Jabosthal and third-order Jabosthal-Lucas sequences and their matrix representations. arXiv 2018, arXiv:1806.03709v1.

20. Didkivska, T.V.; Stopochkina, M.V. Properties of Fibonacci-Narayana numbers. World Math. 2003, 9, $29-36$.

21. Feinberg, M. Fibonacci-tribonacci. Fibonacci Q. 1963, 1, 71-74.

22. Hoggatt, V.E., Jr.; Bicknell, M. Generalized Fibonacci polynomials. Fibonacci Q. 1973, 11, 457-465.

23. Koshy, T. Fibonacci and Lucas Numbers with Applications; John Wiley and Sons Inc.: New York, NY, USA, 2001.

24. Pethe, S. Some identities for tribonacci sequences. Fibonacci Q. 1988, 26, 144-151.

25. Shannon, A.G.; Horadam, A.F. Some properties of third-order recurrence relations. Fibonacci Q. 1972, 10, 135-146.

26. Shannon, A.G.; Anderson, P.G.; Horadam, A.F. Properties of Cordonnier, Perrin and van der Laan numbers. Int. J. Math. Ed. Sci. Technol. 2006, 37, 825-831. [CrossRef]

27. Yalavigi, C.C. Properties of tribonacci numbers. Fibonacci Q. 1972, 10, 231-246.

28. Nurkan, S.K.; Guven, I.A. A note on bicomplex Fibonacci and Lucas numbers. arXiv 2015, arXiv:1508.03972.

29. Aydin, T. Bicomplex Fibonacci quaternions. Chaos Solitons Fractals 2018, 106, 147-153. [CrossRef]

30. Catarino, P. Bicomplex k-Pell Quaternions. Comput. Methods Funct. Theory 2018. [CrossRef] 